Short communication

\title{
Discovery and preliminary SARs of keto-indoles as novel indoleamine 2,3-dioxygenase (IDO) inhibitors
}

\author{
Eduard Dolušićc ${ }^{\mathrm{a}}$, Pierre Larrieu ${ }^{\mathrm{b}}$, Sébastien Blanc ${ }^{\mathrm{c}}$, Frédéric Sapunaric ${ }^{\mathrm{d}}$, Jenny Pouyez ${ }^{\mathrm{a}}$, \\ Laurence Moineaux $^{a}$, Delphine Colette ${ }^{c}$, Vincent Stroobant ${ }^{b}$, Luc Pilotte ${ }^{b}$, Didier Colau ${ }^{b}$, Thierry Ferain ${ }^{c}$, \\ Graeme Fraser $^{c}$, Moreno Galleni ${ }^{d}$, Jean-Marie Frère ${ }^{d}$, Bernard Masereel ${ }^{a}$, Benoît Van den Eynde ${ }^{b}$, \\ Johan Wouters $^{\mathrm{a}}$, Raphaël Frédérick ${ }^{\mathrm{a}, *}$ \\ ${ }^{a}$ Drug Design and Discovery Center, University of Namur, 61 Rue de Bruxelles, 5000 Namur, Belgium \\ ${ }^{\mathrm{b}}$ Ludwig Institute for Cancer Research, Université Catholique de Louvain, 74 Avenue Hippocrate, 1200 Brussels, Belgium \\ ${ }^{\mathrm{c}}$ Euroscreen SA, 47 Rue Adrienne Boland, 6041 Gosselies, Belgium \\ ${ }^{\mathrm{d}}$ Centre d'Ingénierie des Protéines, Université de Liège, Allée du 6 août, 4000 Liège, Belgium
}

\section{A R T I C L E I N F O}

\section{Article history:}

Received 1 December 2010

Received in revised form

17 February 2011

Accepted 21 February 2011

Available online 26 February 2011

Keywords:

Indoleamine 2,3-dioxygenase

Enzyme inhibitors

Keto-indoles

IDO

Structure-based drug discovery

Structure-activity relationships

\begin{abstract}
A B S T R A C T
Indoleamine 2,3-dioxygenase (IDO) is an important new therapeutic target for the treatment of cancer. With the aim of discovering novel IDO inhibitors, a virtual screen was undertaken and led to the discovery of the keto-indole derivative 1a endowed with an inhibitory potency in the micromolar range. Detailed kinetics were performed and revealed an uncompetitive inhibition profile. Preliminary SARs were drawn in this series and corroborated the putative binding orientation as suggested by docking.
\end{abstract}

(c) 2011 Elsevier Masson SAS. All rights reserved.

\section{Introduction}

Immunotherapy is a promising new strategy for cancer therapy. It consists of the therapeutic vaccination of cancer patients to stimulate their (natural) immune system against cancer cells. This approach, however, showed limited efficacy in vivo. Cancer cells are actually able to develop enzymatic mechanisms allowing tumors to resist or escape immune rejection. Among the enzymes involved, indoleamine 2,3-dioxygenase (IDO) plays a key role [1].

IDO is an intracellular monomeric heme-containing enzyme that catalyzes the rapid degradation of tryptophan (Trp) in the initial step of the kynurenine pathway, the de novo biosynthetic route for nicotinamide adenine dinucleotide (NAD) production $[2,3]$. This results in a local Trp depletion that severely affects the proliferation of $\mathrm{T}$ lymphocytes and is thereby profoundly immunosuppressive [4]. Pioneering studies by Munn and Mellor

\footnotetext{
* Corresponding author. Tel.: +32 817242 90; fax: +32 81724238 .

E-mail address: raphael.frederick@fundp.ac.be (R. Frédérick).
}

described that, except for some dendritic cells, IDO is not expressed in normal tissue $[5,6]$. However, it is highly produced in the placenta and is required for the fetus immune tolerance by the organism of the mother during pregnancy [6,7]. Recently, Van den Eynde showed that many types of human tumors express IDO in a constitutive manner [1]. The expression of IDO in human ovarian and colon carcinomas is also associated with poor prognosis and reduced survival $[8,9]$. Finally, because IDO expression is strongly induced by interferon-gamma [10], even tumors that do not constitutively express IDO may do so when exposed to inflammatory conditions, e.g. resulting from an ongoing immune response. IDO thus noticeably protects foreign cells against immune rejection. Based on its implication in immunosuppression, and particularly in cancer tumors, IDO clearly represents an attractive target for the development of inhibitors [11-14].

Until recently, the best known IDO inhibitors [15] displayed affinities in the micromolar range and comprised mainly Trp derivatives such as 1-methyl-L-tryptophan (1MT) [16] $\left(K_{\mathrm{i}} \sim 34 \mu \mathrm{M}\right)$ and $\beta$-carbolines [17]. In 2006, potent nanomolar inhibitors were isolated from marine invertebrate extracts $[18,19]$ At the same time, 
new brassinin-based IDO inhibitors were published [20,21], the best having a $K_{\mathrm{i}}$ of $12 \mu \mathrm{M}$. In 2008, two new classes of compounds were reported based on the earlier discovery of the marine invertebrate inhibitors. Carr et al. identified tryptamine quinone as the core pharmacophore of exiguamine $A$ and described a series of derivatives, the best showing a $K_{\mathrm{i}}$ of $0.2 \mu \mathrm{M}$ [22]. Kumar et al. reported optimization of the naphthoquinone core of annulin $B$ and designed derivatives, partly based on structural modeling, with $\mathrm{IC}_{50}$ values reaching $60 \mathrm{nM}$ [23]. In 1989, 4-phenylimidazole (PIM) was identified as a modestly potent IDO inhibitor [24]. Despite the uncompetitive inhibition kinetics, the authors showed through spectroscopic studies that PIM binds into the active site of IDO. Moreover, they discovered a preferred binding of PIM to the inactive ferric $\left(\mathrm{Fe}^{3+}\right)$ form of the enzyme, which may explain this apparent discrepancy. The first crystal structure of human IDO complexed with the inhibitor reported later [25] confirmed the binding of PIM in the active site. A recently undertaken structurebased study [26] of PIM-derived molecules prompted by Sugimoto's work confirmed the antecedent findings while providing access to novel inhibitors with low micromolar potencies.

Very recently, novel natural product inhibitors $\left(\mathrm{IC}_{50} \approx 2 \mu \mathrm{M}\right)$ [27] and potent competitive inhibitors including a hydroxyamidine chelating motif ( $\mathrm{IC}_{50}$ values up to $60 \mathrm{nM}$ ) have also been reported [28].

Many IDO inhibitors show either noncompetitive or uncompetitive inhibition kinetics similar to those of PIM and norharman, which have been shown to bind directly to the heme iron and to occupy the presumed Trp binding site [26]. The interpretation of IDO inhibition kinetics may, however, be complicated due to the preferential binding of some inhibitors to the inactive ferric form of IDO, and the redox activity of others. Since many of the IDO inhibitors that have been discovered recently might fail in in vivo testing, there is still considerable interest in discovering novel IDO inhibitor families [29].

The two three-dimensional structures of IDO, in complex with PIM and the cyanide ion $\left(\mathrm{CN}^{-}\right)$, provide important data for the structure-based drug design of novel IDO inhibitors [25]. From a structural point of view, IDO is characterized by a small and highly lipophilic active site (Fig. 1a).

In the crystal structure, PIM interacts with the heme iron through its $\mathrm{N}-1$ atom, projecting its phenyl ring toward the lipophilic cavity (Pocket A, Fig. 1b). The PIM binding site consists of residues Tyr126, Cys129, Val130, Phe163, Phe164, Ser167, Leu234, Gly262, Ser263, Ala264, and the heme ring (Fig. 1c). Putative hydrogen bonding sites are the thiol group of Cys129, the hydroxyl group of Ser167, the CO group of Gly262, the NH group of Ala264, and the heme 7-propionate group. Ligands larger than PIM may also interact with Phe226, $\operatorname{Arg} 231$, Ser235, Phe291, Ile354, and Leu384, which are located at the binding site entrance. Here, additional hydrogen bonds are possible with the side chain of Arg231. A hydrophobic pocket in this region is provided by Phe163, Phe226, Leu234, Ile354, and the heme ring (Pocket B, Fig. 1b). The cyanide-bound structure (PDB entry 2D0U) differs from the PIMbound structure mainly in the access to pocket $A$, which is hindered by an inward movement of the backbone of the loop residues 262-266 and the side chain of Phe163, suggesting some flexibility in the active site. In both structures, two buffer molecules (2-[Ncyclohexylamino]ethanesulfonic acid) are bound at the entrance of

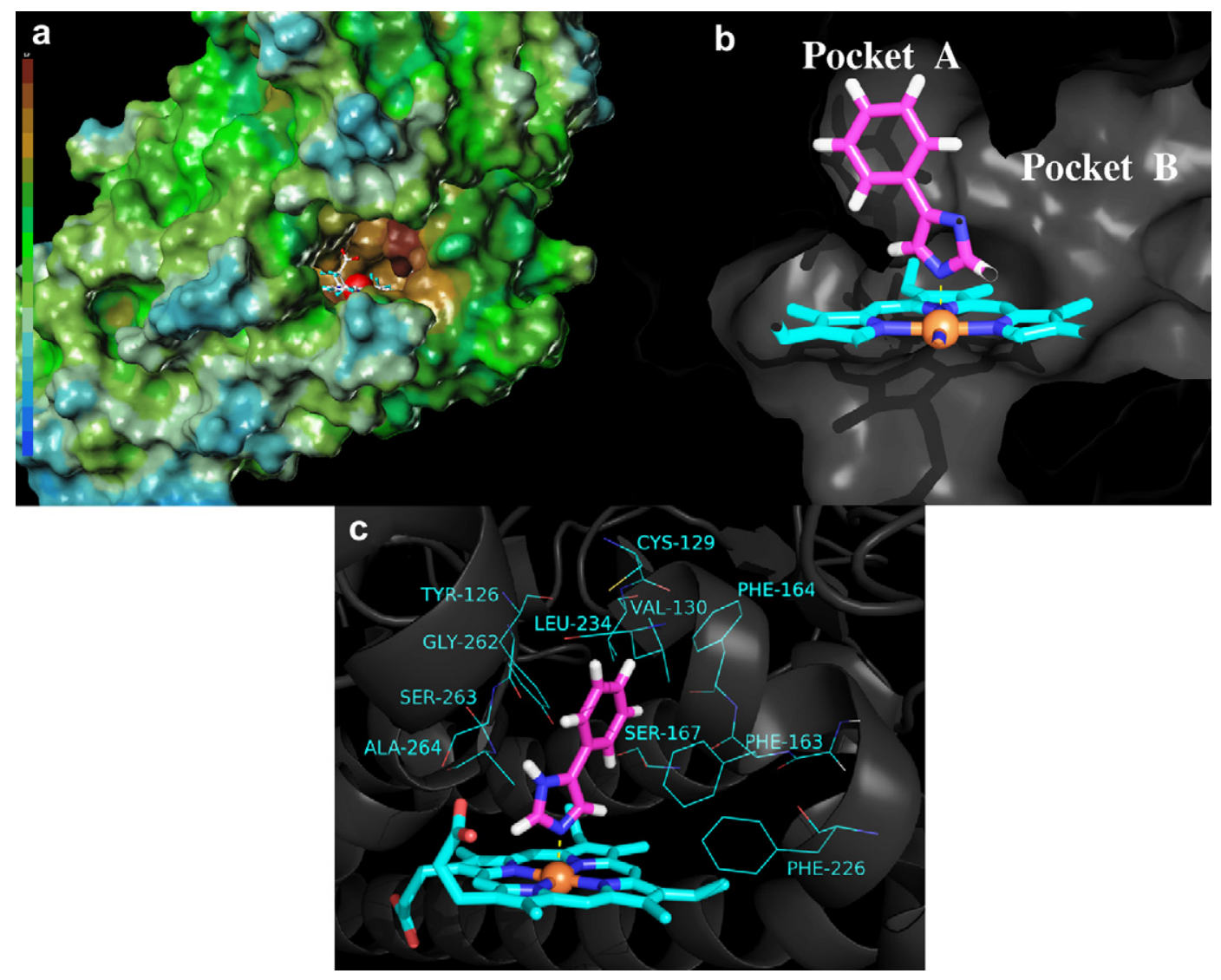

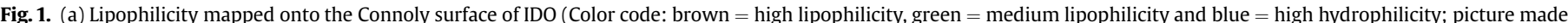

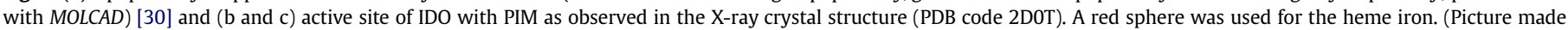
using PYMOL) [31]. (For interpretation of the references to colour in this figure legend, the reader is referred to the web version of this article.) 


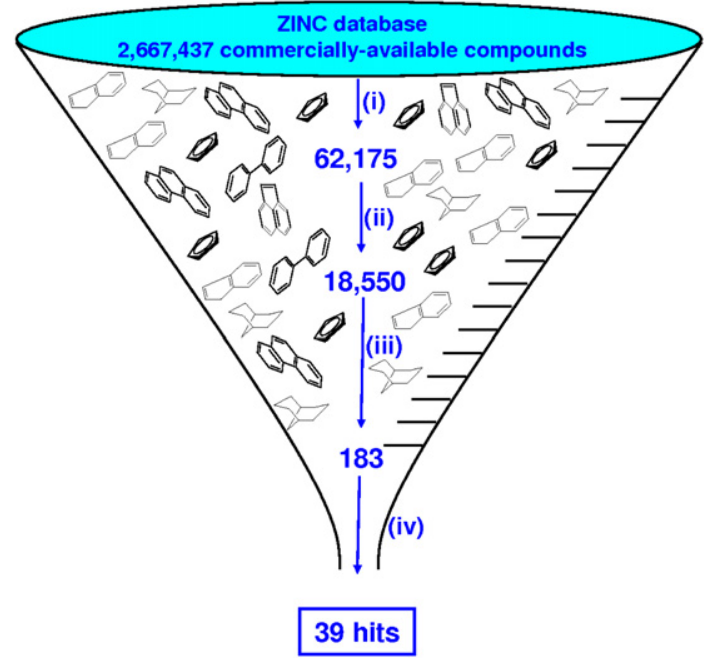

Fig. 2. IDO virtual screening flowchart. (i) Lipinski-style rules, (ii) goldscore $\geq 50$, (iii) Cscore $\geq 4$, (iv) Visual analysis and commercial-availability.

the active site, forming a hydrogen bond to the heme propionate and interacting with pocket $\mathrm{B}$.

As the enzyme active site is now well known, it represents a very good tool to undertake a direct approach of receptor-based drug discovery and design. Among these, virtual screening (VS) [32] involves the computational screening of very large libraries of commercially-available chemicals that complement targets of known structure, followed by the experimental testing of those with the best predicted binding energies. We have applied this strategy in the present work for the discovery of new IDO inhibitors.

\section{Results}

Various chemical libraries, for example the $\mathrm{NCI}$ database (National Cancer Institute), the ACD library (Available Chemical
Directory) and the ZINC database [33] (over 2.5 million commercially-available compounds) can be used to perform VS. We chose the latter as it is free, web-accessible, offers the ligands in a readyto-dock 3D format, and hits are marketed for confirmatory biological evaluation.

Since careful docking of the entire ZINC library would take a prohibitively long time, filters were used to limit the number of compounds to be considered before the docking experiment. The VS flowchart we employed is depicted in Fig. 2.

(i) Lipinski-style rules were first applied [34]. As our primary aim at this very first step was to select only compounds that could serve as leads for future medicinal chemistry development, we used simple molecular descriptors such as the molecular weight (MW $\leq 250$ ), the hydrophobicity $(\log P \leq 2.5)$, and the number of rotatable bonds $(\mathrm{RB} \leq 5)$ as the first filter. Then, all of the qualifying structures were downloaded from the ZINC website and transformed into a UNITY hit list file (Sybyl version 7.3) [30,35] for compatibility with our modeling system. This resulted in a library of roughly 62,000 structures (Fig. 2, (i)).

(ii) All fragments were then docked within the IDO active site (PDB code 2D0T) by means of the automated GOLD program [36]. For each ligand, 3 conformations were generated and ranked according to the GOLDSCORE. Only the conformations possessing the best score were subsequently retained and the top-ranked ligands (GOLDSCORE $\geq 50$ ) were isolated. This led to a set of 18550 structures.

(iii) This set was further refined using Cscore calculations [30]. Cscore is a combination of five different scoring functions, namely G-, D-, PMF-, F- and Chemscore. The Cscore value is an integer comprised between 5 (all of the five scoring function have identified the ligand as a good potential binder) and 0 (none of the 5 scoring functions has identified the ligand as a binder) and helps distinguishing the best potential ligands from the less probable ones. Concomitantly to Cscore evaluation, the individual contributions (van der Waals, H-bonding, etc.) of Chemscore were also analyzed and particularly the

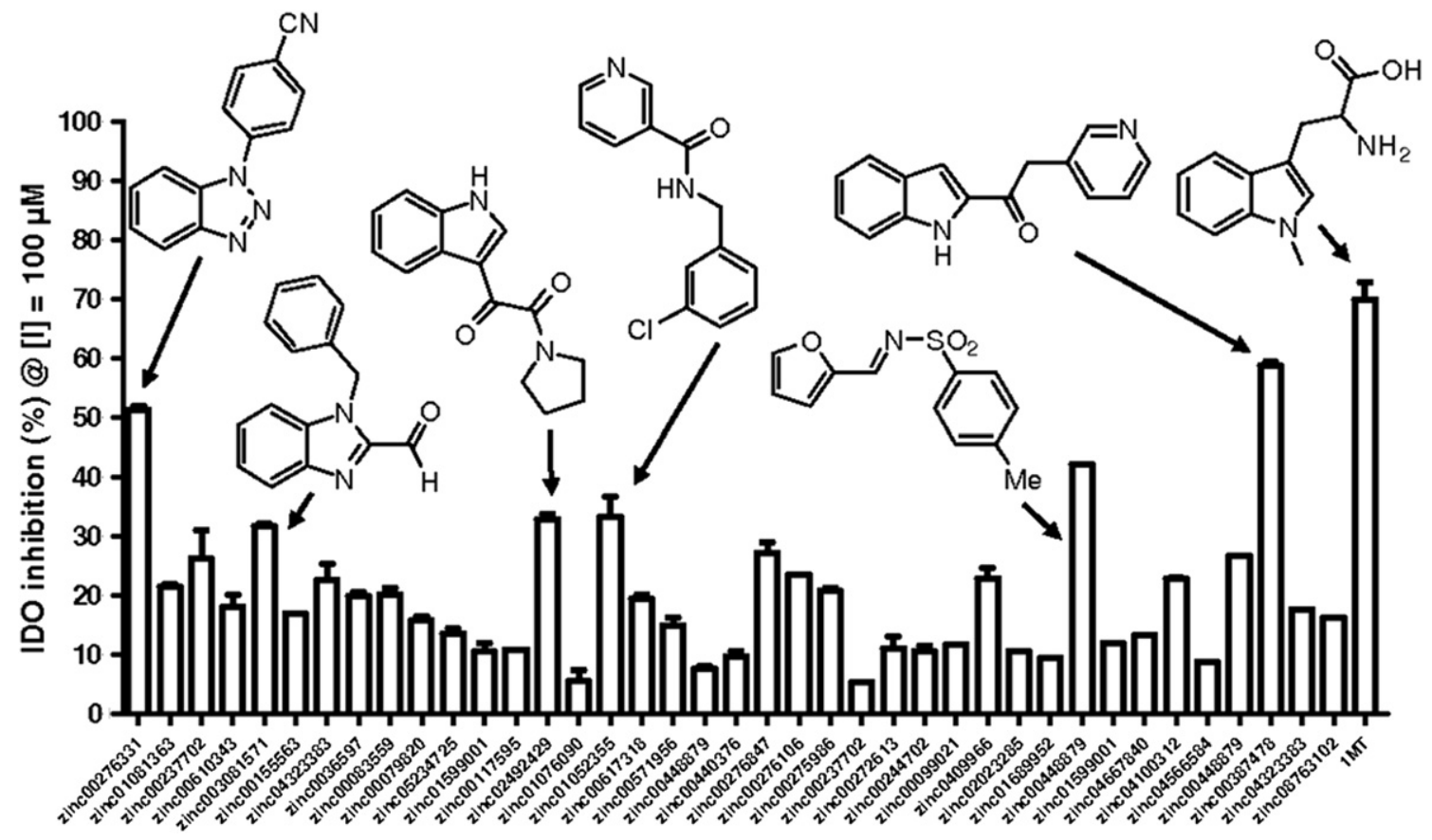

Fig. 3. IDO inhibition of the selected compounds at $[\mathrm{I}]=100 \mu \mathrm{M}$. 
Table 1

Inhibitory IDO activity of 1a identified using VS.

\begin{tabular}{lccl}
\hline Cmpd & $\mathrm{IC}_{50}(\mu \mathrm{M})$ & $K i(\mu \mathrm{M})$ & Type of inhibition \\
\hline 1MT & $100 \pm 20$ & 7 & Competitive \\
Zinc00387478 = 1a & $65 \pm 7$ & 190 & Uncompetitive \\
\hline
\end{tabular}

chemscore-metal value which reflects the formation of an interaction between the ligand and the metal, namely, the heme iron. Thus, keeping compounds characterized by a Cscore value $\geq 4$ and a chemscore-metal value of 1 , respectively, led to a final set of 183 derivatives.

(iv) These 183 structures were visually analyzed within the IDO active site and 39 of them were selected based on structural diversity, synthetic accessibility, appropriate conformation and real commercial availability. This step introduces some subjectivity in the selection and relies on the expertise of the medicinal chemist(s) who perform it. The 39 structures were obtained from the vendors (Chembridge, Sigma, Specs, Maybridge, Acros).

The biological activity of the 39 compounds was evaluated using a colorimetric in vitro IDO inhibition assay [1]. Briefly, the inhibitors are incubated $\left(6 \mathrm{~min}, 37^{\circ} \mathrm{C}\right)$ with IDO and L-Trp, the natural substrate. Trichloroacetic acid (TCA) is then added to quench the reaction and the $\mathrm{N}$-formylkynurenine generated is hydrolyzed to kynurenine during the next $30 \mathrm{~min}$. After that time, $p$-dimethylaminobenzaldehyde ( $p D M A B$ ) is added to the reaction mixture to form a Schiff base with kynurenine. The IDO residual activity is measured at $490 \mathrm{nM}$ and corresponds to the Schiff base formed.

Six derivatives (Zinc code 276331, 3081571, 2492429, 1052355, 448979 , and 387478 ) out of the 39 compounds analyzed displayed an inhibitory potency $>30 \%$ at a concentration of $100 \mu \mathrm{M}$, two of them (Zinc 276331 and Zinc 387478) possessing more than 50\% IDO inhibition at this concentration (Fig. 3).

Unfortunately, the low solubility of the benzotriazole (Zinc00276331) did not allow determination of a proper IC $_{50}$ for this compound. In contrast, the indole derivative (Zinc00387478 = 1a) proved to be at least as potent as $1 \mathrm{MT}$, being characterized by an $\mathrm{IC}_{50}$ of $65 \mu \mathrm{M}$ for IDO inhibition (Table 1 ). This compound is characterized by an indole nucleus substituted in the 2-position by a 3-pyridinylethanone function. Interestingly, no biological activity was reported so far for this compound, making it an interesting tool for chemical optimization to further enhance IDO inhibition. Compared to 1MT, 1a also has the advantage of containing no chiral center.

Detailed kinetic experiments were performed on 1a to elucidate its mode of inhibition. In contrast to $1 \mathrm{MT}$ which shows a competitive IDO inhibition profile (Table 1), this compound exhibited an uncompetitive inhibition mode (Fig. 4). It is characterized by an inhibition constant $\left(K_{\mathrm{i}}\right)$ of $\sim 190 \mu \mathrm{M}$ (Table 1$)$, in a range similar to the $\mathrm{IC}_{50}$. These data indicate that, as an uncompetitive inhibitor, 1a would only bind to the substrate-bound enzyme. This might be surprising taking into account the way it was discovered i.e. virtual screening/docking to the heme substrate IDO binding site. However, recently Kumar et al. observed similar inhibition kinetic profiles for phenylimidazoles [26], despite their binding to the heme iron at the active site as experimentally established through X-ray studies [25]. One hypothesis that could, at least in part, explain this uncompetitive profile would be a high affinity of these molecules to the inactive ferric vs. active ferrous form of the enzyme. Similar hypotheses were also reported earlier for $\beta$-carboline derivatives and could probably explain the kinetic profile observed for 1a [17]. To corroborate our hypothesis, we performed UV analysis of IDO with or without 1a and analyzed the effect on the Soret band, characteristic of the heme group. The optical absorption spectrum of the substrate-free ferric IDO exhibits a Soret maximum at $404 \mathrm{~nm}$ and the binding of L-Trp causes, as reported previously, a red-shift of the Soret maximum to $410 \mathrm{~nm}$ together with a hypochromic effect (Fig. 5a) [37]. In the case of 1a addition (Fig. 5b), a dose-dependent hypochromic effect without red-shift is observed thus confirming a close interaction of 1a with the heme binding site.

Next, preliminary SARs around 1a were investigated. Indeed, one attracting feature of virtual screening is that it allows, once a hit is identified, to directly analyze the interactions stabilizing the molecule within the active site cavity of the target, and subsequently to suggest pharmacomodulations on the basis of the putative binding orientation. Thus, the interactions stabilizing 1a inside the IDO binding cleft were analyzed in detail and revealed that the oxygen atom of the ketone function, being situated $\sim 2 \AA$ above the plane of the heme, coordinates the heme iron. The indole ring is found to be stabilized in the so-called lipophilic A-pocket of IDO. Indeed, this stabilization of the indole in pocket $A$ was already suggested by other groups to explain substrate binding as well as interaction of 1MT. In this position, the 3-pyridyl group is projected toward the entrance of the active site and is stabilized in the aromatic pocket B through T-shape interactions with Phe163 and Phe226 (Fig. 6). In this orientation, excellent complementarities are found between the indole nucleus and pocket $A$. Indeed, apart from the 4-, and 5-position as well as the 1-position (indole $\mathrm{NH}$ ), only little space is left available to accommodate substituents (Fig. 6).

A few analogs of 1a were then synthesized in order to assess whether or not the binding mode suggested by docking could explain preliminary experimental SARs and thus profitably offer up hypotheses for further structure-based design effort. According to
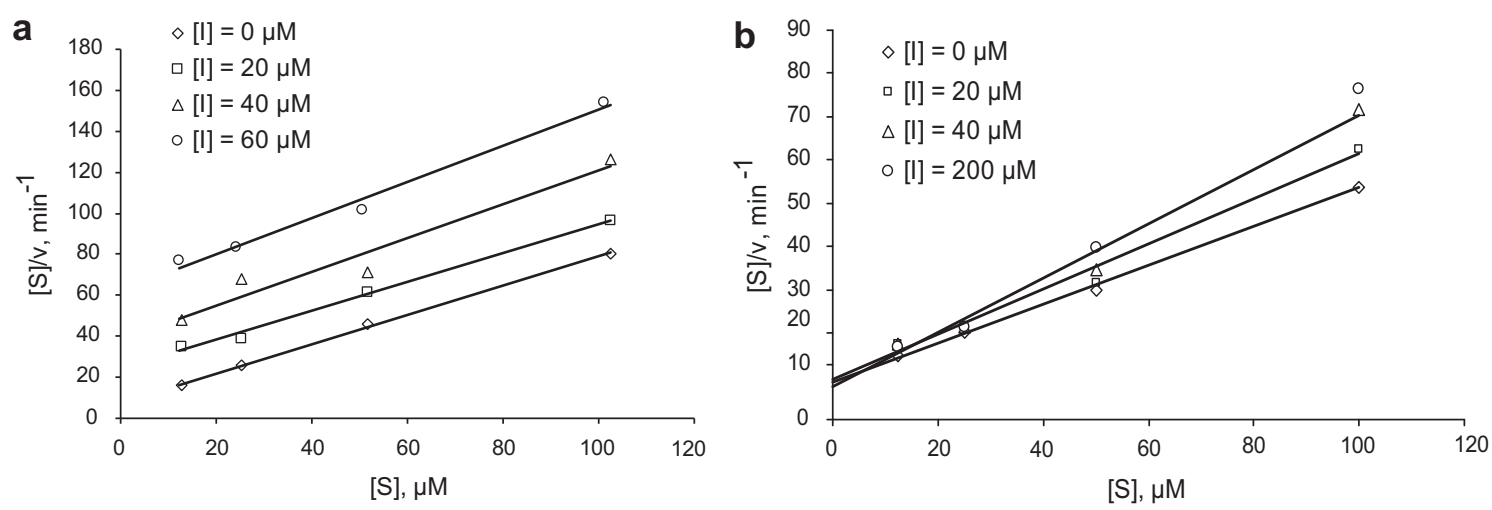

Fig. 4. Hanes plot of IDO activity against L-tryptophan in presence of increasing concentration of 1-methyl-L-tryptophan (1MT) (a) and 1a (b). 

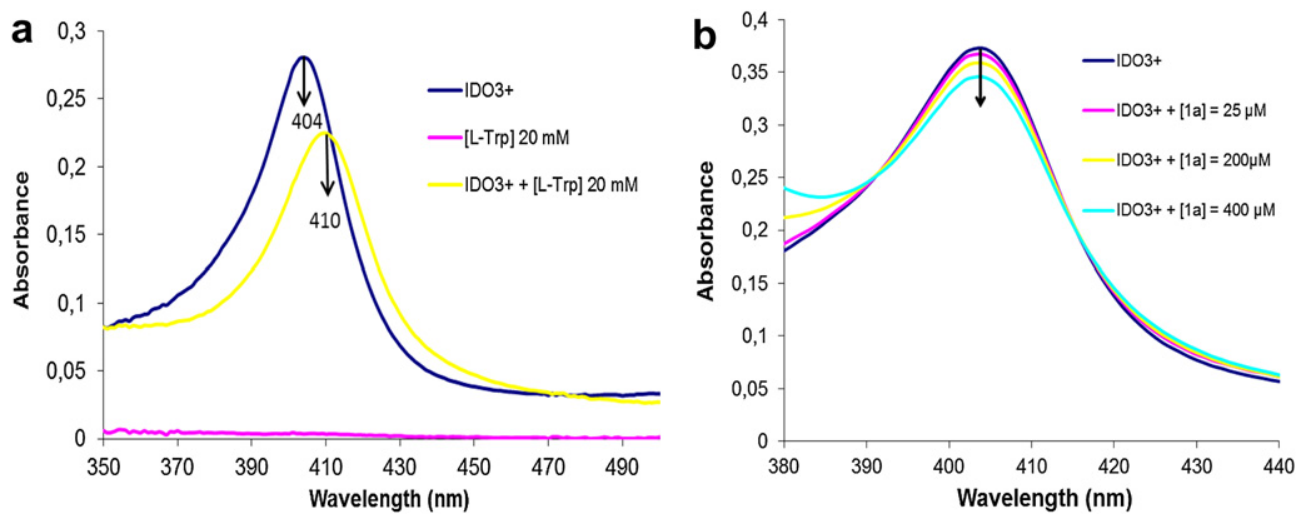

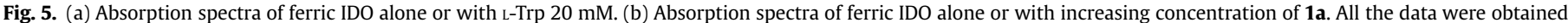
with $12.6 \mu \mathrm{M}$ IDO in $50 \mathrm{mM}$ Tris buffer $(\mathrm{pH} 8.0)$ at room temperature.

the structural analysis reported above, we hypothesized that substitution of the indole in the 1-, 4- and 5-position through introduction of small groups such as halogens, methyl, methoxy or nitro moieties could improve the IDO inhibitory potency in this series. We also investigated the importance of the ketone function of 1a suggested to be directly involved in the interaction with the heme iron, through replacement of the ethanone linker by an ethylene, a hydroxyethylene or an amide.

A synthetic pathway allowing to access these structures was therefore elaborated (Scheme 1). It involves the deprotonation of 3-methylpyridine in presence of lithium diisopropylamide (LDA). The carbanion generated in situ then reacts with a reactive indole carbonyl compound at $0{ }^{\circ} \mathrm{C}$ to provide the desired keto-indole compounds of the general formula $\mathbf{1}$ The Wolff-Kishner reduction and the catalytic hydrogenation of $\mathbf{1 a}\left(R=R^{\prime}=H\right)$, respectively afforded the ethylene linked compound $\mathbf{2}$ and secondary alcohol $\mathbf{3}$. Finally, amide 4 was made from the corresponding $1 \mathrm{H}$-indole-2carboxylic acid by reaction with thionyl chloride followed by nucleophilic substitution with 3-aminopyridine (Scheme 1).

The $\mathrm{IC}_{50}$ 's of the novel indole derivatives were determined on isolated enzyme and are summarized in Table 2.

As expected from our docking analysis, a two-fold improvement in the IDO inhibitory potency is observed when fluorine is introduced in the 5-position (1c) around the indole scaffold. In contrast, its introduction in the 4-position (1b) appears to be detrimental. A bulkier halogen in this 5-position such as a chlorine (1d) also led to a potent IDO inhibitor. The 5-methyl (1e) and the 5-methoxy (1f) analogs are still active but their IDO inhibition is not improved compared to the unsubstituted compound 1a. Finally, the 5-nitro derivative $\mathbf{1 g}$ is deprived of any IDO inhibitory potency. This preliminary SARs suggests, in agreement with the docking study reported above, that only rather small and lipophilic groups are tolerated in this position.

Regarding the importance of the ketone function on 1a, replacement of the ethanone linker with an ethylene (2) or even a hydroxyl-ethylene spacer (3) totally suppresses the IDO inhibitory potency thus confirming the importance of the ketone pharmacophore for IDO inhibition in this series. Only its replacement with an amide (4) afforded a compound that is still active against IDO.

We also appraised the ability of our derivatives to inhibit tryptophan degradation and kynurenine production in cells expressing murine IDO. The compounds were also screened for their inhibitory activity in cells expressing murine tryptophan 2,3dioxygenase (TDO), an enzyme functionally related to IDO, with a view to investigate their selectivity. For all cell lines, cell viability was evaluated at the end of the assay. This cellular assay is informative for drug development as it evaluates not only the IDO/ TDO inhibitory effect of the compounds but also their capacity to permeate the cell, their potential cytotoxicity, inhibition of tryptophan transporters, and the effects of their metabolites. Concerning the ability of these derivatives to inhibit tryptophan degradation in cells expressing murine IDO, 1c, 1d and $\mathbf{1 e}$ are the most potent derivatives with $20-25 \%$ inhibition of tryptophan degradation at $20 \mu \mathrm{M}$, most of the other compounds being deprived of any cellular activity. The relative discrepancy in IDO inhibition between the isolated enzyme and cellular assays probably reflects a poor permeation in this series. Interestingly, the more potent IDO inhibitors in cell-based assay are deprived of

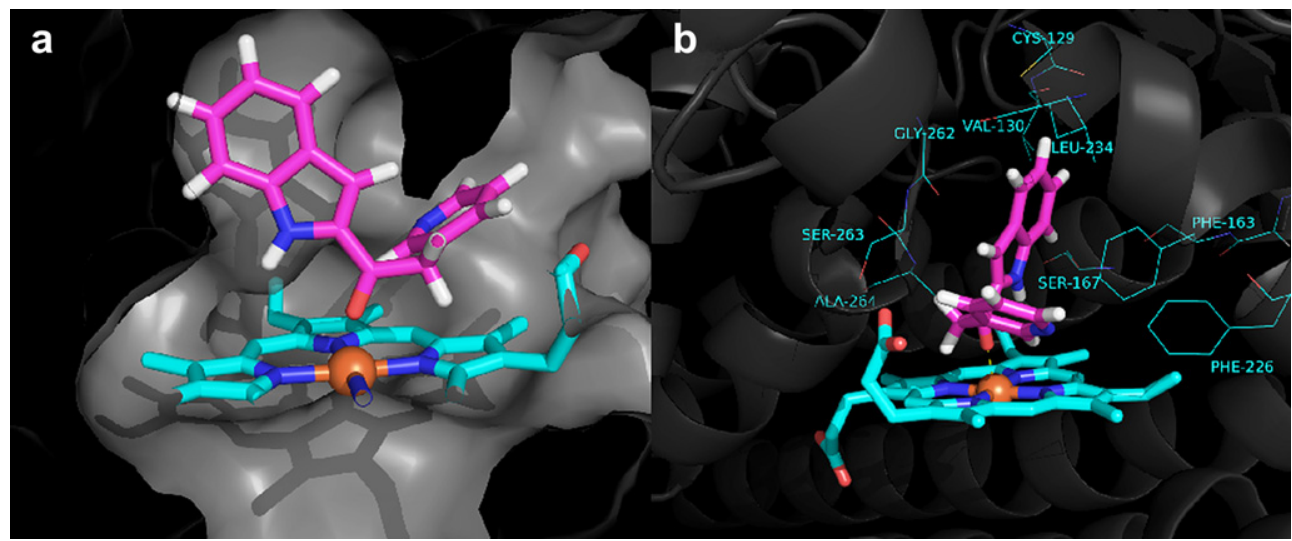

Fig. 6. ( $\mathrm{a}$ and $\mathrm{b}$ ) Predicted binding mode for 1a as docked in GOLD (Pictures made with MOLCAD). 


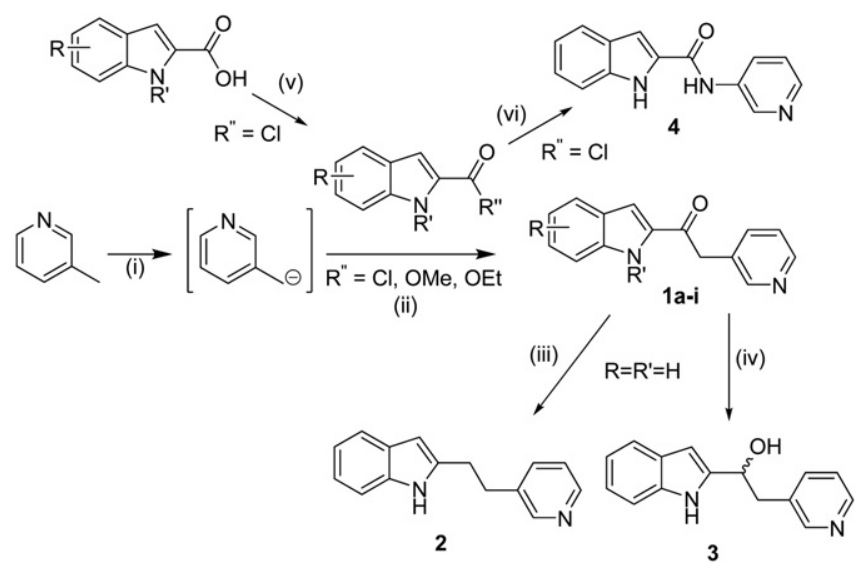

Scheme 1. (i), LDA, THF/hexane, -78 to $0{ }^{\circ} \mathrm{C}, 1 \mathrm{~h}$; (ii) THF/hexane, $0{ }^{\circ} \mathrm{C}$ to rt, $16-24 \mathrm{~h}$; (iii), $\mathrm{H}_{2} \mathrm{NNH}_{2}, \mathrm{KOH},\left(\mathrm{CH}_{2} \mathrm{OH}\right)_{2}, \mu \mathrm{W}, 1$ h then $\mathrm{H}_{2} \mathrm{O} / \mathrm{NH}_{4} \mathrm{Cl}$; (iv) $\mathrm{HCO}_{2} \mathrm{NH}_{4}$, Pd black, $\mathrm{MeOH}$, rt, 3 days; (v) SOCl $, \Delta, 15 \mathrm{~min}$; (vi) 3-aminopyridine, DIPEA, THF, $0^{\circ} \mathrm{C} \rightarrow \mathrm{rt}, 30 \mathrm{~min}$.

any cellular activity on TDO (Table 2), suggesting some selectivity in this series.

In conclusion, a virtual screening strategy combining various filters including high-throughput docking was used to search for new IDO inhibitors. From the 39 final compounds identified and assayed, six derivatives displayed an inhibitory potency $>30 \%$ at a concentration of $100 \mu \mathrm{M}$, two of them possessing more than $50 \%$ IDO inhibition at this concentration. Detailed kinetics revealed an uncompetitive inhibition profile for the best inhibitor 1a. Its binding mode inside the IDO binding cleft was evaluated by means of docking and revealed essential features responsible for the IDO inhibition potency in this series. Preliminary SARs around 1a corroborated this putative binding orientation and support the interest in this series for further drug design effort.

\section{Experimental section}

\subsection{Chemistry}

Details of synthetic procedures used to prepare compounds 1a-i, 2, 3 and $\mathbf{4}$ as well as structural and analytical characterizations can be found in Dolušić et al. [38].

\subsection{General synthetic procedure for compounds $1 \mathbf{1 a}-\mathbf{i}$ [39]}

An oven-dried flask was purged with argon while hot, then allowed to cool down to room temperature under argon and charged with dry THF ( $2 \mathrm{~mL} / \mathrm{mmol}$ of starting indole) and diisopropylamine (4.0 equivalents). The solution was cooled to $-78{ }^{\circ} \mathrm{C}$ in a dry ice/isopropanol bath and $n$-butyl lithium, $1.6 \mathrm{M}$ solution in hexanes ( 4.0 equivalents), was added to give a bright yellow solution. The mixture was stirred for 30 min below $-60^{\circ} \mathrm{C}$ whereupon a solution of picoline (4.0 equivalents) in THF ( $2 \mathrm{~mL} / \mathrm{mmol}$ of starting indole) was added to give a yellow colored mixture. During a further $30 \mathrm{~min}$, the bath temperature was allowed to rise to approx. $0{ }^{\circ} \mathrm{C}$ during which time the reaction mixture became an orange suspension. The temperature was then kept at $0{ }^{\circ} \mathrm{C}$ (using an ice/water bath) for an additional $30 \mathrm{~min}$. At this point, a solution of the indole-2-carbonyl compound (1.0 equivalent) in THF ( $4 \mathrm{~mL} /$ mmol) was added dropwise over the flask wall. The reaction mixture darkened (dark purple to dark brown color in most cases) and was then allowed to attain room temperature overnight. At $18 \mathrm{~h}$ after the addition of the indole component, the reaction was quenched with saturated aqueous ammonium chloride solution (10 $\mathrm{mL} / \mathrm{mmol}$ of starting compound) and stirred for several minutes. Upon partition between ethyl acetate and water, the aqueous phase was extracted twice with ethyl acetate and the combined organic layers were dried over $\mathrm{MgSO}_{4}$. The title products were purified by chromatography eluting with a gradient of ethyl acetate in cyclohexane (the products typically eluted with 70-80\% of ethyl acetate). The products were then precipitated by concentration of the pooled column fractions combined with the addition of cyclohexane. The precipitates formed were filtered off, washed twice with cyclohexane and dried at $40{ }^{\circ} \mathrm{C}$ in vacuo to yield analytically pure samples.

\subsection{Biology}

\subsubsection{Protein expression and purification of recombinant human IDO}

The coding region for human IDO (Ala2-Gly403) was cloned into a derivative of plasmid pET9 (Novagen). The recombinant plasmid, pETIDO, encodes a histidine tag at the N-terminus of IDO. Bacterial strain BL21 AI (Invitrogen) was used for overexpression of IDO and transformed with the pETIDO plasmid. The transformed cells were grown on a rotary shaker at $37^{\circ} \mathrm{C}$ and $220 \mathrm{rpm}$ to an OD600 of 1.2, in LB medium supplemented with $25 \mu \mathrm{g} / \mathrm{mL}$ kanamycin, $50 \mu \mathrm{g} / \mathrm{mL}$ L-tryptophan and $10 \mu \mathrm{M}$ bovine hemin (Sigma). The culture was

Table 2

Biological activity of the indole derivatives $1 a-i, 2,3,4$.

\begin{tabular}{|c|c|c|c|c|c|c|}
\hline Compound & $\mathrm{R}$ & $\mathrm{R}^{\prime}$ & $\begin{array}{l}\text { IDO IC }_{50} \\
(\mu \mathrm{M})\end{array}$ & $\begin{array}{l}\text { IDO cell assay } \\
\text { inhibition \% } \\
@ 20 \mu \mathrm{M}^{\mathrm{a}}\end{array}$ & $\begin{array}{l}\text { TDO cell assay } \\
\text { inhibition \% } \\
@ 20 \mu \mathrm{M}^{\mathrm{a}}\end{array}$ & $\begin{array}{l}\text { Cell viability } \\
@ 20 \mu \mathrm{M}(\%)\end{array}$ \\
\hline $1 \mathrm{MT}$ & & & $100 \pm 20$ & 20 & $\mathrm{NI}$ & No toxicity \\
\hline $1 \mathbf{a}$ & $\mathrm{H}$ & $\mathrm{H}$ & $65 \pm 7$ & 13 & $\mathrm{NI}$ & No toxicity \\
\hline 1b & $4-\mathrm{F}$ & $\mathrm{H}$ & $153 \pm 23$ & 12 & $\mathrm{NI}$ & 80 \\
\hline 1c & $5-\mathrm{F}$ & $\mathrm{H}$ & $36.0 \pm 0.1$ & 24 & $\mathrm{NI}$ & No toxicity \\
\hline 1d & $5-\mathrm{Cl}$ & $\mathrm{H}$ & $24.6 \pm 0.1$ & 24 & $\mathrm{NI}$ & 90 \\
\hline 1e & 5-Me & $\mathrm{H}$ & $87 \pm 11$ & 21 & $\mathrm{NI}$ & No toxicity \\
\hline 1f & 5-MeO & $\mathrm{H}$ & $49 \pm 1$ & NI & $\mathrm{NI}$ & No toxicity \\
\hline $1 \mathrm{~g}$ & $5-\mathrm{NO}_{2}$ & $\mathrm{H}$ & $\mathrm{NI}$ & NI & $\mathrm{NI}$ & 90 \\
\hline $1 \mathrm{~h}$ & $\mathrm{H}$ & $\mathrm{Me}$ & $34 \pm 2$ & $\mathrm{NI}$ & $\mathrm{NI}$ & No toxicity \\
\hline 1i & $5-\mathrm{F}$ & $\mathrm{Me}$ & $92 \pm 15$ & NI & $\mathrm{NI}$ & 95 \\
\hline 2 & & & $\mathrm{NI}$ & $\mathrm{NI}$ & $\mathrm{NI}$ & 90 \\
\hline$\overline{3}$ & & & NI & NI & $\mathrm{NI}$ & 90 \\
\hline 4 & & & $94 \pm 2$ & $\mathrm{NI}$ & $\mathrm{NI}$ & 75 \\
\hline
\end{tabular}

$\mathrm{NI}=$ no inhibition.

a The indicated values are the result of two independent determinations; variation between experiments is no more than $\pm 5 \%$. 
cooled in a water/ice bath and supplemented again with $50 \mu \mathrm{g} / \mathrm{mL}$ L-tryptophan and $10 \mu \mathrm{M}$ bovine hemin. The expression of Histagged IDO was induced by the addition of $1 \%(\mathrm{w} / \mathrm{v})$ arabinose. Induced cells were grown at $20^{\circ} \mathrm{C}$ and $60 \mathrm{rpm}$ for $20 \mathrm{~h}$. Cells ( $1 \mathrm{~L}$ culture) were collected by centrifugation, resuspended in $40 \mathrm{~mL}$ of $25 \mathrm{mM}$ Mes, $150 \mathrm{mM} \mathrm{KCl}, 10 \mathrm{mM}$ imidazole, and protease inhibitors (complete EDTA free, Roche Applied Science) (pH 6.5), and disrupted with a French press. The extract was clarified by centrifugation and filtration on a $0.22 \mu \mathrm{m}$ filter. The enzyme was purified by IMAC using Ni2p as a ligand and an IMAC HITRAP column ( $5 \mathrm{~mL}, \mathrm{GE}$ Healthcare). Briefly, the extract was loaded on the column with $25 \mathrm{mM}$ Mes, $150 \mathrm{mM} \mathrm{KCl}$, and $10 \mathrm{mM}$ imidazole ( $\mathrm{pH} \mathrm{6.5).} \mathrm{The}$ column was washed with $50 \mathrm{~mL}$ of the same buffer with the imidazole concentration adjusted to $100 \mathrm{mM}$. Finally, the protein was eluted with $25 \mathrm{mM}$ Mes, $150 \mathrm{mM} \mathrm{KCl}$, and $50 \mathrm{mM}$ EDTA (pH 6.5). The buffer was then exchanged into $25 \mathrm{mM}$ Mes and $150 \mathrm{mM}$ $\mathrm{KCl}$ (pH 6.5) using a HITRAP desalting column (GE Healthcare). The purity of the enzyme was estimated to be $>95 \%$ based on the SDSPAGE gel and Coomassie blue staining. The ratio of absorbance at $404 \mathrm{~nm}$ to that at $280 \mathrm{~nm}$ of the protein was around 1.9.

\subsubsection{Enzymatic assay}

The enzymatic inhibition assays were performed as described by Takikawa et al. [40] with some modifications. Briefly, the reaction mixture $(200 \mu \mathrm{L})$ contained potassium phosphate buffer (50 mM, pH 6.5) ascorbic acid (10 mM), methylene blue $(5 \mu \mathrm{M})$, purified recombinant IDO $(43 \mu \mathrm{M})$, L-Trp $(100 \mu \mathrm{M})$, and DMSO $(10 \mu \mathrm{L})$. The inhibitors were serially diluted 10 -fold from 1000 to $0.1 \mu \mathrm{M}$ in pure DMSO or, if not soluble at $1000 \mu \mathrm{M}$, by four orders of magnitude from their highest soluble concentration. The reaction was conducted at $37{ }^{\circ} \mathrm{C}$ for $6 \mathrm{~min}$ and stopped by addition of $30 \%$ $(\mathrm{w} / \mathrm{v})$ trichloroacetic acid $(40 \mu \mathrm{L})$. To convert $N$-formylkynurenine to kynurenine, the tubes were incubated at $37{ }^{\circ} \mathrm{C}$ for $30 \mathrm{~min}$, followed by centrifugation at $20000 \mathrm{~g}$ for $20 \mathrm{~min}$. Finally, $150 \mu \mathrm{L}$ of supernatant is added to $150 \mu \mathrm{L}$ of $p$-dimethylaminobenzaldehyde $(p \mathrm{DMAB})(2 \%, v / v)$ in acetic acid to generate a Schiff base with kynurenine that was detected at a wavelength of $480 \mathrm{~nm}$.

\subsubsection{Cellular tests}

Cell Line. A plasmid construct encoding murine IDO was transfected into mouse mastocytoma line P815B. Clone P185B-mIDO clone 6 [1], which overexpresses IDO, was selected and used for the cellular assay. Mouse IDO shares a $62 \%$ sequence identity with human IDO, and the active site residues are $100 \%$ conserved. For the assays evaluating inhibition of mouse TDO, a plasmid construct encoding murine TDO was transfected into mouse mastocytoma cell line P815B [1]. Clone P815B-mTDO clone 12, which overexpresses TDO, was selected and used for the cellular assay. Mouse TDO shares $88 \%$ sequence identity with human TDO, and the active site residues are $100 \%$ conserved.

Assay. The assay was performed in 96-well flat bottom plates seeded with $2 \times 10^{5}$ cells in a final volume of $200 \mu$ l. To determine whether compounds were significant IDO or TDO inhibitors, the cells were incubated overnight at $37{ }^{\circ} \mathrm{C}$ in HBSS (Hanks Balanced Salt Solution, Invitrogen) supplemented with $80 \mu \mathrm{M}$ L-Tryptophan and $20 \mu \mathrm{M}$ of the compound. The plates were then centrifuged $10 \mathrm{~min}$ at $300 \mathrm{~g}$, and $150 \mu \mathrm{l}$ of the supernatant were collected. The supernatant was analyzed by HPLC to measure the concentration of tryptophan and kynurenine, based on the retention time and the UV absorption ( $280 \mathrm{~nm}$ for tryptophan, $360 \mathrm{~nm}$ for kynurenine). For the HPLC analysis, $50 \mu \mathrm{l}$ of supernatant were mixed with $500 \mu \mathrm{l}$ acetonitrile to precipitate the proteins. After centrifugation, the supernatant was collected, concentrated on a speedvac, resuspended in a final volume of $100 \mu \mathrm{l}$ water and injected in the HPLC (C18 column). In those conditions, about $50 \%$ of the initial amount of tryptophan was degraded in the absence of inhibitor, and an equimolar amount of kynurenine was produced. The percentages of inhibition of tryptophan degradation and kynurenine production by the compounds were calculated in reference to this maximal activity. The initial wells containing the cells in the remaining volume of $50 \mu \mathrm{l}$ were used to estimate cell viability in a classical MTT assay. To that end, $50 \mu \mathrm{l}$ of culture medium (Iscove medium with $10 \%$ FCS and amino acids) were added to the wells together with $50 \mu \mathrm{l}$ of MTT. After $3-4 \mathrm{~h}$ of incubation at $37^{\circ} \mathrm{C}, 100 \mu \mathrm{l}$ of SDS/ DMF were added to dissolve the crystals of formazan blue and the absorbance at $570 \mathrm{~nm} / 650 \mathrm{~nm}$ was measured after overnight incubation at $37^{\circ} \mathrm{C}$.

\subsubsection{Steady states kinetics and inhibition studies}

Human IDO with an N-terminal His tag was expressed in Escherichia coli and purified to homogeneity as described by Oda [41]. The enzymatic reactions were conducted at $37{ }^{\circ} \mathrm{C}$ for $12 \mathrm{~min}$ in $50 \mathrm{mM}$ potassium phosphate $\mathrm{pH} 6.5$, neutralized ascorbic acid $10 \mathrm{mM}$, methylene blue $5 \mu \mathrm{M}$, DMSO 5\%, catalase 100 units per $\mathrm{ml}$, L-tryptophan and $8.5 \mathrm{nM}$ IDO. The reaction was initiated by addition of IDO. At various intervals ( $2 \mathrm{~min})$, aliquots $(200 \mu \mathrm{L})$ were removed, mixed with $40 \mu \mathrm{l}$ of trichloroacetic acid (30\% w/v), and incubated at $65{ }^{\circ} \mathrm{C}$ for $10 \mathrm{~min}$ to achieve the conversion of $\mathrm{N}$-formylkynurenine to kynurenine. The final L-tryptophan concentrations varied between $12.5 \mu \mathrm{M}$ and $100 \mu \mathrm{M}$. The concentration of L-tryptophan was determined using the absorption coefficient $\epsilon_{280}=5500 \mathrm{M}^{-1} \mathrm{~cm}^{-1}$ and the substrate was filtered through a membrane filter prior to each experiment (pore size, $0.22 \mu \mathrm{m}$ ). The spectrophotometer method to measure kynurenine with the Ehrlich reagent was carried out according to Takikawa [40] and the initial rates were calculated from the absorbance increase at $480 \mathrm{~nm}\left(\epsilon_{480}=15820 \mathrm{M}^{-1} \mathrm{~cm}^{-1}\right)$ [42]. Each experiment was performed at least three times. The initial rates were fit to the Michaelis-Menten equation by nonlinear regression analysis using Graphpad prism software. Apparent $K m$ and $K i$ values were determined by varying the concentrations of the substrate and the inhibitors. The mode of inhibition was determined by plotting [S]/v against [S] (Hanes plot) and 1/v against [I] (Dixon plot).

Spectroscopic measurements. Optical absorption spectra were recorded on a Specord 50 photometer (Analytik Jena). All experiments were performed in $50 \mathrm{mM}$ Tris- $\mathrm{HCl}$ buffer $(\mathrm{pH} 8.0)$ at $25^{\circ} \mathrm{C}$.

\subsection{Molecular modeling}

Molecular modeling studies were carried out on a Linux workstation. The compounds were downloaded from the ZINC website (http://zinc.docking.org) and transformed into a UNITY hit list by mean of SYBYL (version 8.0) [30]. Docking was performed using the 3D-coordinates of IDO ( $\mathrm{pdb}$ code 2DOT) with the help of the automated GOLD program [36] (active site definition: residues within $7 \AA$ around phenylimidazole) using parameters especially designed for virtual screening experiments (7-8 fold acceleration). The hits that were obtained after ranking using GOLDSCORE and CSCORE were re-docked for confirmatory evaluation and their geometry subsequently optimized using the MINIMIZE module. The minimization process uses the Powell method with the Tripos force field (dielectric constant $1 r$ ) to reach a final convergence of $0.01 \mathrm{kcal} \mathrm{mol}^{-1}$.

\section{Acknowledgments}

This work is supported by the FNRS (Belgium) and the Walloon region (CANTOL grant no 5678). R.F. is greatly indebted to the Belgian "Fonds de la Recherche Scientifique-FNRS" for the award of a postdoctoral research grant. 


\section{References}

[1] C. Uyttenhove, L. Pilotte, I. Theate, V. Stroobant, D. Colau, N. Parmentier, T. Boon, B.J. Van den Eynde, Evidence for a tumoral immune resistance mechanism based on tryptophan degradation by indoleamine 2,3-dioxygenase, Nat. Med. 9 (2003) 1269-1274.

[2] S.A. Rafice, N. Chauhan, I. Efimov, J. Basran, E.L. Raven, Oxidation of L-tryptophan in biology: a comparison between tryptophan 2,3-dioxygenase and indoleamine 2,3-dioxygenase, Biochem. Soc. Trans. 37 (2009) 408-412.

[3] M. Sono, M.P. Roach, E.D. Coulter, J.H. Dawson, Heme-containing oxygenases, Chem. Rev. 96 (1996) 2841-2888.

[4] P. Hwu, M.X. Du, R. Lapointe, M. Do, M.W. Taylor, H.A. Young, Indoleamine 2,3-dioxygenase production by human dendritic cells results in the inhibition of T cell proliferation, J. Immunol. 164 (2000) 3596-3599.

[5] A.L. Mellor, D.H. Munn, IDO expression by dendritic cells: tolerance and tryptophan catabolism, Nat. Rev. Immunol. 4 (2004) 762-774.

[6] D.H. Munn, M. Zhou, J.T. Attwood, I. Bondarev, S.J. Conway, B. Marshall, C. Brown, A.L. Mellor, Prevention of allogeneic fetal rejection by tryptophan catabolism, Science 281 (1998) 1191-1193.

[7] Y. Kudo, C.A. Boyd, Human placental indoleamine 2,3-dioxygenase: cellular localization and characterization of an enzyme preventing fetal rejection, Biochim. Biophys. Acta 1500 (2000) 119-124.

[8] G. Brandacher, A. Perathoner, R. Ladurner, S. Schneeberger, P. Obrist, C. Winkler, E.R. Werner, G. Werner-Felmayer, H.G. Weiss, G. Gobel, R. Margreiter, A. Konigsrainer, D. Fuchs, A. Amberger, Prognostic value of indoleamine 2,3-dioxygenase expression in colorectal cancer: effect on tumor-infiltrating T cells, Clin. Cancer Res. 12 (2006) 1144-1151.

[9] A. Okamoto, T. Nikaido, K. Ochiai, S. Takakura, M. Saito, Y. Aoki, N. Ishii, N. Yanaihara, K. Yamada, O. Takikawa, R. Kawaguchi, S. Isonishi, T. Tanaka, M. Urashima, Indoleamine 2,3-dioxygenase serves as a marker of poor prognosis in gene expression profiles of serous ovarian cancer cells, Clin. Cancer Res. 11 (2005) 6030-6039.

[10] B.S. Leung, L.E. Stout, E.G. Shaskan, R.M. Thompson, Differential induction of indoleamine-2,3-dioxygenase (IDO) by interferon-gamma in human gynecologic cancer cells, Cancer Lett. 66 (1992) 77-81.

[11] A.J. Muller, J.B. DuHadaway, P.S. Donover, E. Sutanto-Ward, G.C. Prendergast, Inhibition of indoleamine 2,3-dioxygenase, an immunoregulatory target of the cancer suppression gene Bin1, potentiates cancer chemotherapy, Nat Med. 11 (2005) 312-319.

[12] A.J. Muller, W.P. Malachowski, G.C. Prendergast, Indoleamine 2,3-dioxygenase in cancer: targeting pathological immune tolerance with small-molecule inhibitors, Expert Opin. Ther. Targets 9 (2005) 831-849.

[13] A.J. Muller, G.C. Prendergast, Marrying immunotherapy with chemotherapy: why say IDO? Cancer Res. 65 (2005) 8065-8068.

[14] A.J. Muller, G.C. Prendergast, Indoleamine 2,3-dioxygenase in immune suppression and cancer, Curr. Cancer Drug Targets 7 (2007) 31-40.

[15] A. Macchiarulo, E. Camaioni, R. Nuti, R. Pellicciari, Highlights at the gate of tryptophan catabolism: a review on the mechanisms of activation and regulation of indoleamine 2,3-dioxygenase (IDO), a novel target in cancer disease, Amino Acids 37 (2009) 219-229.

[16] S.G. Cady, M. Sono, 1-Methyl-dl-tryptophan, beta-(3-benzofuranyl)-dl-alanine (the oxygen analog of tryptophan), and beta-[3-benzo(b)thienyl]-dl-alanine (the sulfur analog of tryptophan) are competitive inhibitors for indoleamine 2,3-dioxygenase, Arch. Biochem. Biophys. 291 (1991) 326-333.

[17] N. Eguchi, Y. Watanabe, K. Kawanishi, Y. Hashimoto, O. Hayaishi, Inhibition of indoleamine 2,3-dioxygenase and tryptophan 2,3-dioxygenase by beta-carboline and indole derivatives, Arch. Biochem. Biophys. 232 (1984) 602-609.

[18] H.C. Brastianos, E. Vottero, B.O. Patrick, R. Van Soest, T. Matainaho, A.G. Mauk, R.J. Andersen, Exiguamine A, an indoleamine-2,3-dioxygenase (IDO) inhibitor isolated from the marine sponge Neopetrosia exigua, J. Am. Chem. Soc. 128 (2006) 16046-16047.

[19] A. Pereira, E. Vottero, M. Roberge, A.G. Mauk, R.J. Andersen, Indoleamine 2,3dioxygenase inhibitors from the Northeastern Pacific Marine Hydroid Garveia annulata, J. Nat. Prod. 69 (2006) 1496-1499.

[20] T. Banerjee, J.B. Duhadaway, P. Gaspari, E. Sutanto-Ward, D.H. Munn, A.L. Mellor, W.P. Malachowski, G.C. Prendergast, A.J. Muller, A key in vivo antitumor mechanism of action of natural product-based brassinins is inhibition of indoleamine 2,3-dioxygenase, Oncogene 27 (2008) 2851-2857.

[21] P. Gaspari, T. Banerjee, W.P. Malachowski, A.J. Muller, G.C. Prendergast, J. DuHadaway, S. Bennett, A.M. Donovan, Structure-activity study of brassinin derivatives as indoleamine 2,3-dioxygenase inhibitors, J. Med. Chem. 49 (2006) 684-692.
[22] G. Carr, M.K. Chung, A.G. Mauk, R.J. Andersen, Synthesis of indoleamine 2,3dioxygenase inhibitory analogues of the sponge alkaloid exiguamine A, J. Med. Chem. 51 (2008) 2634-2637.

[23] S. Kumar, W.P. Malachowski, J.B. DuHadaway, J.M. LaLonde, P.J. Carroll, D. Jaller, R. Metz, G.C. Prendergast, A.J. Muller, Indoleamine 2,3-dioxygenase is the anticancer target for a novel series of potent naphthoquinone-based inhibitors, J. Med. Chem. 51 (2008) 1706-1718.

[24] M. Sono, S.G. Cady, Enzyme kinetic and spectroscopic studies of inhibitor and effector interactions with indoleamine 2,3-dioxygenase. 1. Norharman and 4phenylimidazole binding to the enzyme as inhibitors and heme ligands, Biochemistry 28 (1989) 5392-5399.

[25] H. Sugimoto, S. Oda, T. Otsuki, T. Hino, T. Yoshida, Y. Shiro, Crystal structure of human indoleamine 2,3-dioxygenase: catalytic mechanism of $\mathrm{O} 2$ incorporation by a heme-containing dioxygenase, Proc. Natl. Acad. Sci. U S A 103 (2006) 2611-2616.

[26] S. Kumar, D. Jaller, B. Patel, J.M. LaLonde, J.B. DuHadaway, W.P. Malachowski, G.C. Prendergast, A.J. Muller, Structure based development of phenylimidazole-derived inhibitors of indoleamine 2,3-dioxygenase, J. Med. Chem. 51 (2008) 4968-4977.

[27] G. Carr, W. Tay, H. Bottriell, S.K. Andersen, A.G. Mauk, R.J. Andersen, Plectosphaeroic acids $\mathrm{A}, \mathrm{B}$, and $\mathrm{C}$, indoleamine 2,3-dioxygenase inhibitors produced in culture by a marine isolate of the fungus Plectosphaerella cucumerina, Org. Lett. 11 (2009) 2996-2999.

[28] E.W. Yue, B. Douty, B. Wayland, M. Bower, X. Liu, L. Leffet, Q. Wang, K.J. Bowman, M.J. Hansbury, C. Liu, M. Wei, Y. Li, R. Wynn, T.C. Burn, H.K. Koblish, J.S. Fridman, B. Metcalf, P.A. Scherle, A.P. Combs, Discovery of potent competitive inhibitors of indoleamine 2,3-dioxygenase with in vivo pharmacodynamic activity and efficacy in a mouse melanoma model, J. Med. Chem. 52 (2009) 7364-7367.

[29] U.F. Rohrig, L. Awad, A. Grosdidier, P. Larrieu, V. Stroobant, D. Colau, V. Cerundolo, A.J. Simpson, P. Vogel, B.J. Van den Eynde, V. Zoete, O. Michielin, Rational design of indoleamine 2,3-dioxygenase inhibitors, J. Med. Chem. 53 (2010) 1172-1189.

[30] Sybyl 8.0, Tripos Inc., in, 1699 South Hanley Rd., St. Louis, Missouri, 63144, USA.

[31] W.L. Delano, The PyMOL Molecular Graphics System on World Wide Web.http://pymol.sourceforge.net/ (accessed 08.02.11).

[32] B.K. Shoichet, Virtual screening of chemical libraries, Nature 432 (2004) $862-865$.

[33] J.J. Irwin, B.K. Shoichet, ZINC-a free database of commercially available compounds for virtual screening, J. Chem. Inf. Model. 45 (2005) 177-182.

[34] S.J. Teague, A.M. Davis, P.D. Leeson, T. Oprea, The design of leadlike combinatorial libraries, Angew. Chem. Int. Ed. Engl. 38 (1999) 3743-3748.

[35] R.D. Clark, A. Strizhev, J.M. Leonard, J.F. Blake, J.B. Matthew, Consensus scoring for ligand/protein interactions, J. Mol. Graph Model. 20 (2002) 281-295.

[36] G. Jones, P. Willett, R.C. Glen, A.R. Leach, R. Taylor, Development and validation of a genetic algorithm for flexible docking, J. Mol. Biol. 267 (1997) 727-748.

[37] C. Lu, Y. Lin, S.R. Yeh, Spectroscopic studies of ligand and substrate binding to human indoleamine 2,3-dioxygenase, Biochemistry 49 (2010) 5028-5034.

[38] E. Dolušić, P. Larrieu, S. Blanc, F. Sapunaric, B. Norberg, L. Moineaux, D. Colette, V. Stroobant, L. Pilotte, D. Colau, T. Ferain, G. Fraser, M. Galeni, J.M. Frere, B. Masereel, B. Van den Eynde, J. Wouters, R. Frederick, Indol-2-yl ethanones as novel indoleamine 2,3-dioxygenase (IDO) inhibitors, Bioorg. Med. Chem. 19 (2011) 1550-1561.

[39] R.G. Sundberg, J.G. Luis, R.L. Parton, S. Schreiber, P.C. Srinivasan, P. Lamb, P. Forcier, R.B. Bryan, Chloroacetamide photocyclization of indole derivatives. synthesis, stereochemistry, and crystal structure of 3,7-methano-3-azacycloundecino5[,4-blindole (deethylquebrachamine) derivatives, J. Org. Chem. 23 (1978) 4859-4865.

[40] O. Takikawa, T. Kuroiwa, F. Yamazaki, R. Kido, Mechanism of interferongamma action. Characterization of indoleamine 2,3-dioxygenase in cultured human cells induced by interferon-gamma and evaluation of the enzymemediated tryptophan degradation in its anticellular activity, J. Biol. Chem. 263 (1988) 2041-2048.

[41] S. Oda, H. Sugimoto, T. Yoshida, Y. Shiro, Crystallization and preliminary crystallographic studies of human indoleamine 2,3-dioxygenase, Acta Crystallogr. Sect. F Struct. Biol. Cryst Commun. 62 (2006) 221-223.

[42] E. Alegre, A.S. Lopez, A. Gonzalez, Tryptophan metabolites interfere with the Ehrlich reaction used for the measurement of kynurenine, Anal. Biochem. 339 (2005) 188-189. 